\title{
LA-UR-12-24551
}

Approved for public release; distribution is unlimited.

Title:

Author(s):

Intended for:

\section{Modeling of Non-Precious Metal Catalyst Active Sites}

Holby, Edward F.

Taylor, Christopher D.

Invited Talk

\section{- LOSAlamos}

\author{
EST. 1943
}

Disclaimer:

Los Alamos National Laboratory, an affirmative action/equal opportunity employer,is operated by the Los Alamos National

Security, LLC for the National NuclearSecurity Administration of the U.S. Department of Energy under contract DE-AC52-06NA25396.

By approving this article, the publisher recognizes that the U.S. Government retains nonexclusive, royalty-free license to

publish or reproduce the published form of this contribution, or to allow others to do so, for U.S. Government purposes.

Los Alamos National Laboratory requests that the publisher identify this article as work performed under the auspices of the

U.S. Departmentof Energy. Los Alamos National Laboratory strongly supports academic freedom and a researcher's right to publish; as an institution, however, the Laboratory does not endorse the viewpoint of a publication or guarantee its technical correctness. 


\title{
Modeling of Non-Precious Metal Catalyst Active Sites
}

\author{
Edward (Ted) F. Holby and Chris Taylor \\ Los Alamos National Lab \\ Energy Technology Seminar \\ September $10^{\text {th }}, 2012$
}




\section{Talk Outline}

- Background and Motivation

- Monte Carlo Search

- Insights from Density Functional Theory

- Vibrational spectra comparison with experiment

- Defected graphene nanoribbon stability

- Oxygen reduction reaction pathways

- Conclusions and Future Work 


\section{Talk Outline}

- Background and Motivation

- Monte Carlo Search

- Insights from Density Functional Theory

- Vibrational spectra comparison with experiment

- Defected graphene nanoribbon stability

- Oxygen reduction reaction pathways

- Conclusions and Future Work 


\section{Why Fuel Cells?}

- Low temperature fuel cells (PEFC)

- Replacement for internal combustion engine (ICE)

- Potentially removes need for fossil fuels

- Reduction of energy imports

- Enables renewable technologies

- Highly efficient ( 2x ICE), high power density

- Clean point-of-use

- No smog, GHG, NOX, etc.
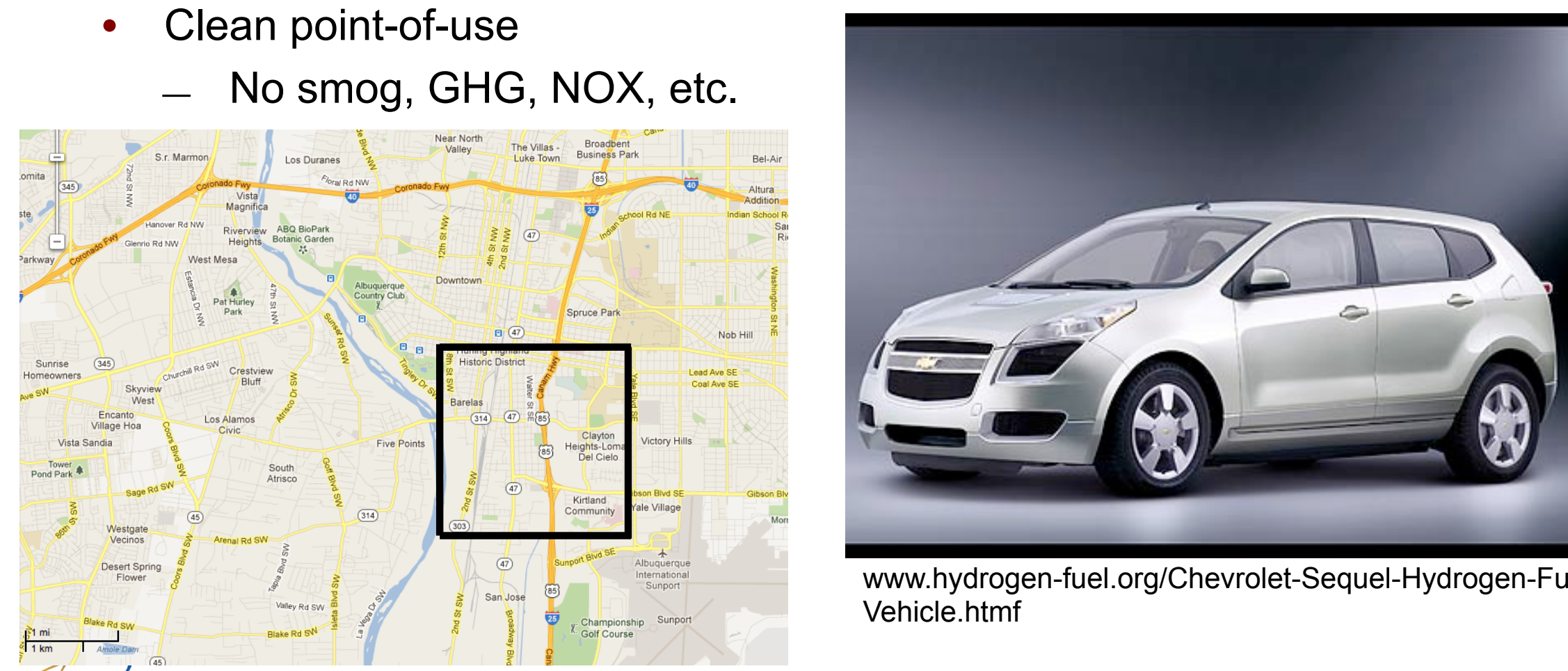

www.hydrogen-fuel.org/Chevrolet-Sequel-Hydrogen-Fuel-CellVehicle.htmf 


\section{How a Fuel Cell Works}

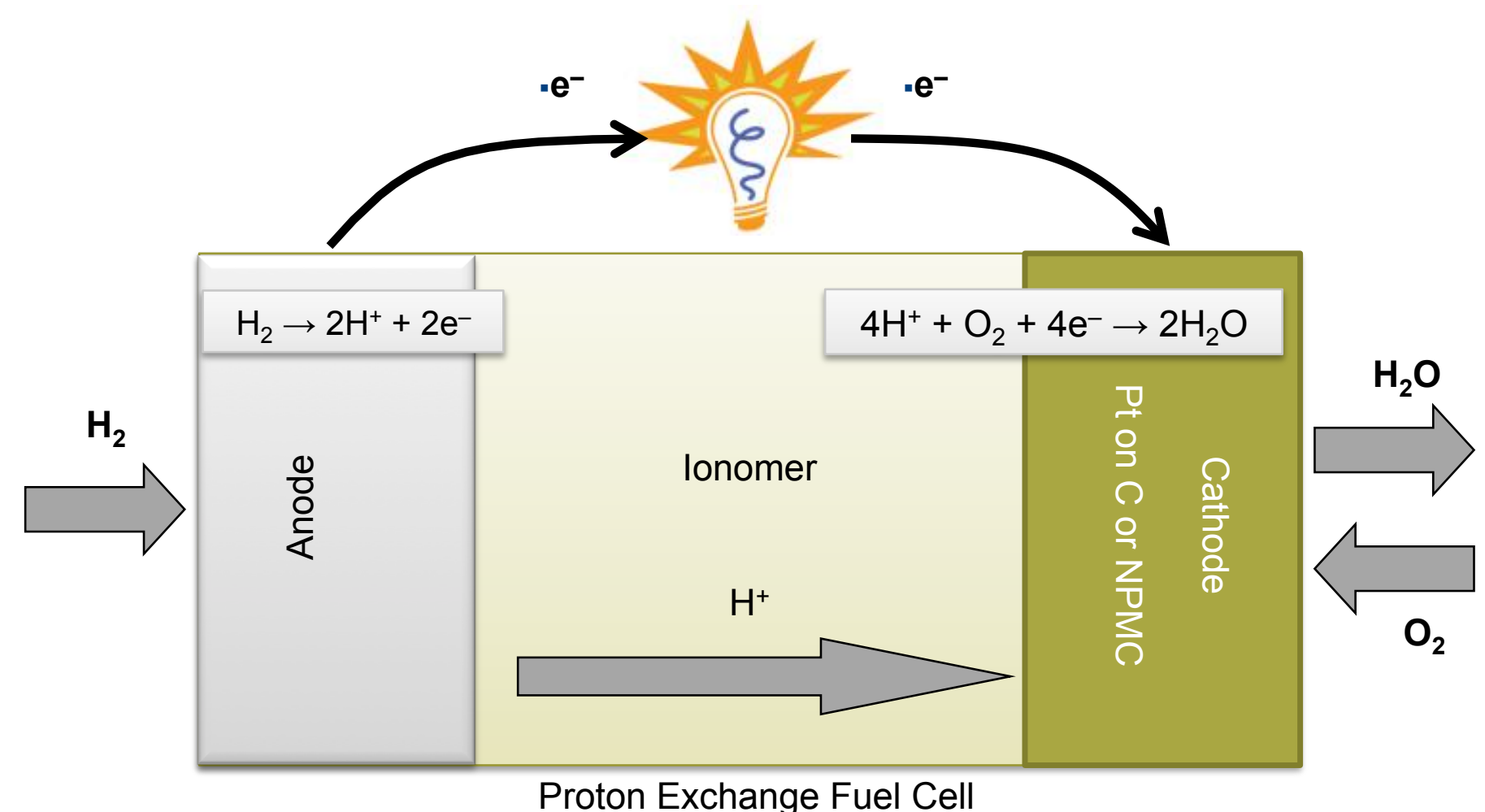

Proton Exchange Fuel Cell 


\section{The Problem with Platinum}

- Pt and Pt based alloys - main ingredient in state of art ORR catalysts

- Issues with Pt: cost, supply, and durability

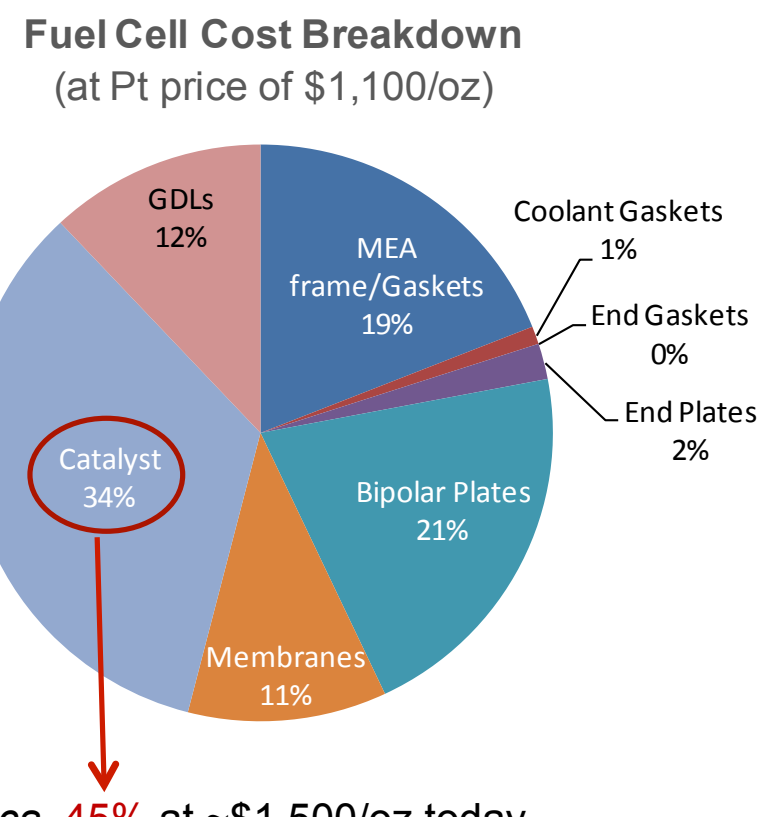

ca. $45 \%$ at $\sim \$ 1,500 /$ oz today (a)

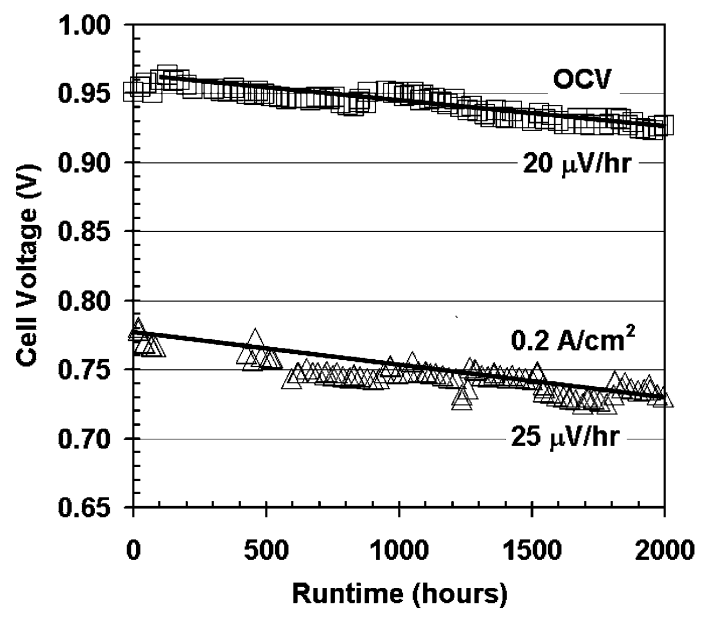

(b)

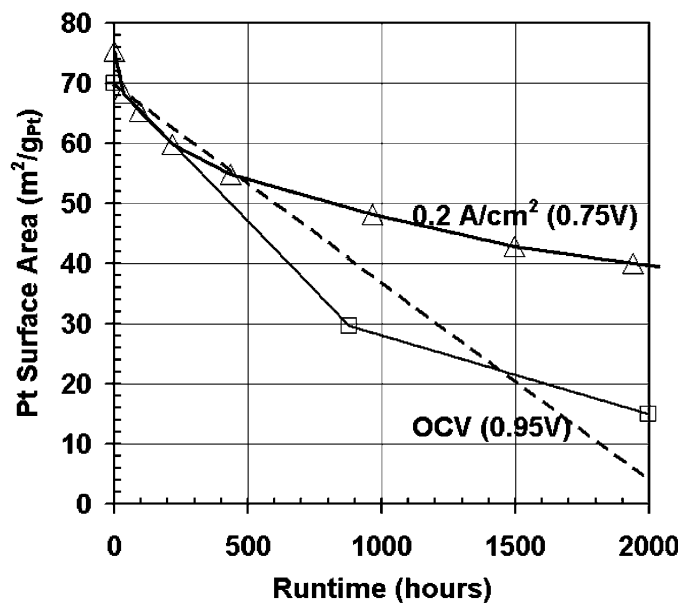

Ferreira, et al., J. Electrochm. Soc. (2005).

James, et al., 2010 DOE Hydrogen Program Review (2010).Revised P. Zelenay 


\section{Solutions for Platinum Problems}

- Solutions:

- Use less Pt

- Find Pt replacement

- Non-precious metal catalysts (NPMCs) -- earth-abundant materials to catalyze ORR

- Chalcogenides

- Oxides

- Nitrides

- Macrocycles

- M-N/C structures

- Activity/Stability main issues

- State of art NPMC developed at LANL (MPA-11)

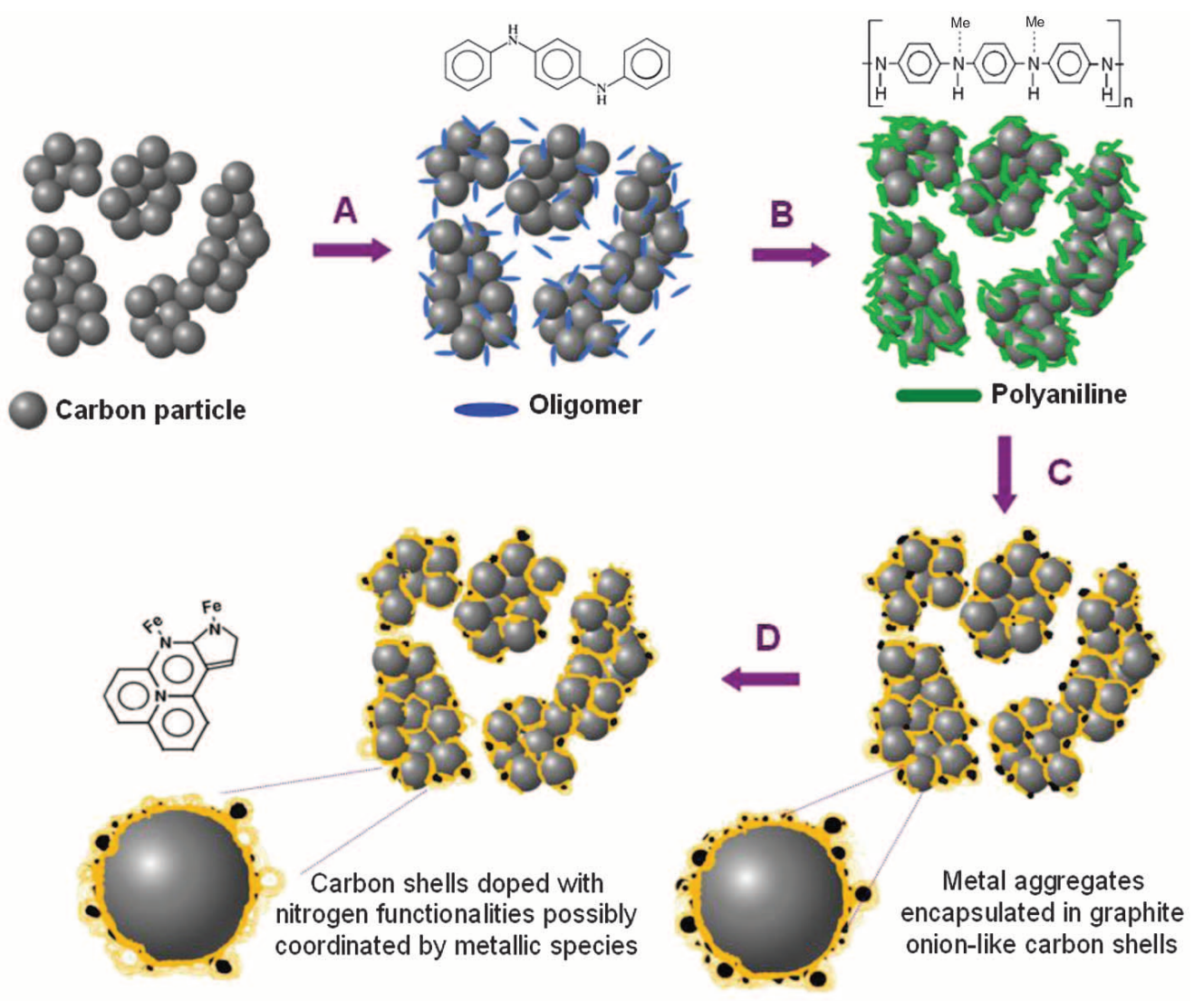

Wu, et al., Science (2011). 


\section{How Modeling Can Help}

- Active site structure $=$ rational design principle

$\rightarrow$ Maximize number of active sites / activity

$\rightarrow$ Maximize stability

- What is atomic scale structure of active site?

- Amorphous $\mathrm{C}$ host (pyrolyzed) with $\mathrm{N}$ and transition metal (Fe/Co) dopants - many structures!

- Is Fe/Co part of active site or simply template?

- Active debate ongoing in community

- Can we do better?

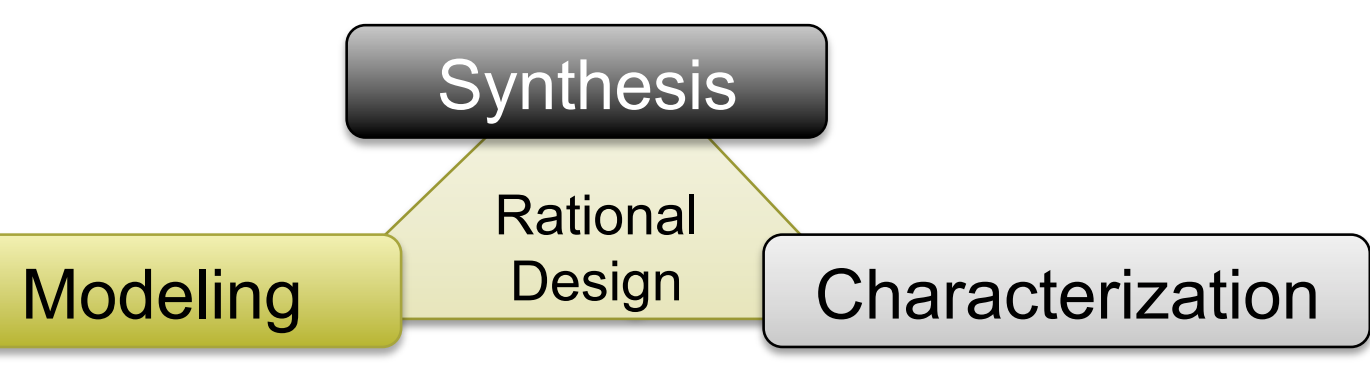




\section{Proposed Active Sites}
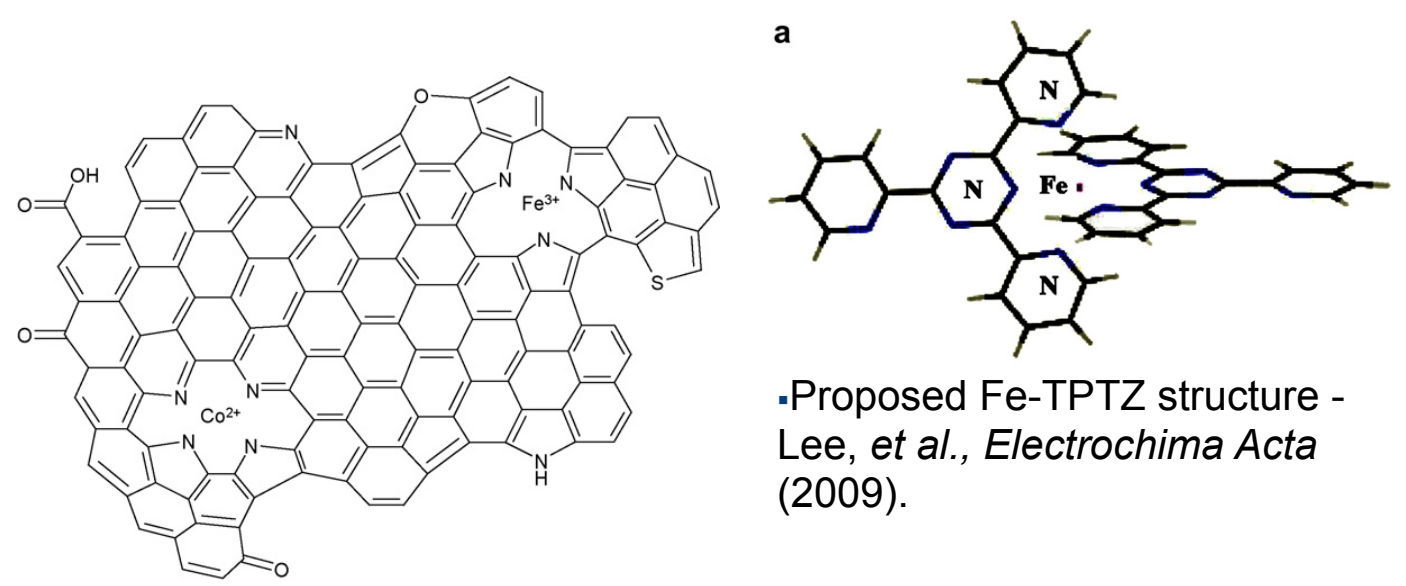

-Proposed Fe-TPTZ structure Lee, et al., Electrochima Acta (2009).

-Proposed Fe-N/C structure Tributsch, et al., Electrochima Acta (2008).

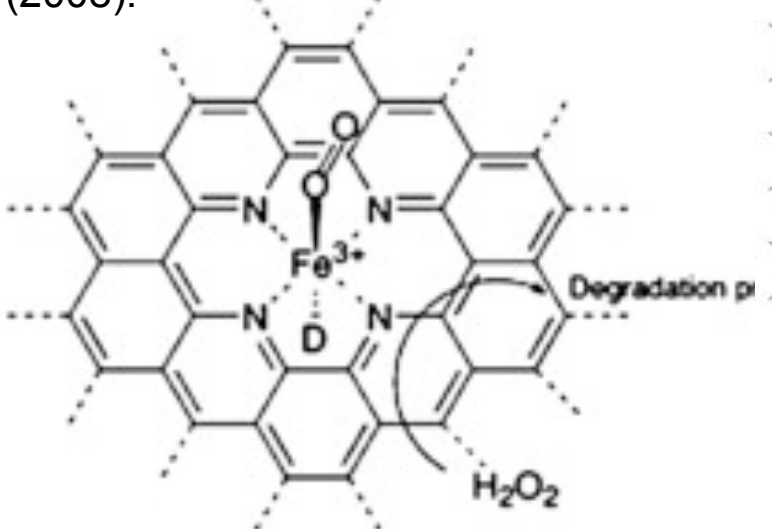

.Proposed Fe-TMPPC structure -

Schulenburg, et al., J. Phys. Chem. B

(2003)
D

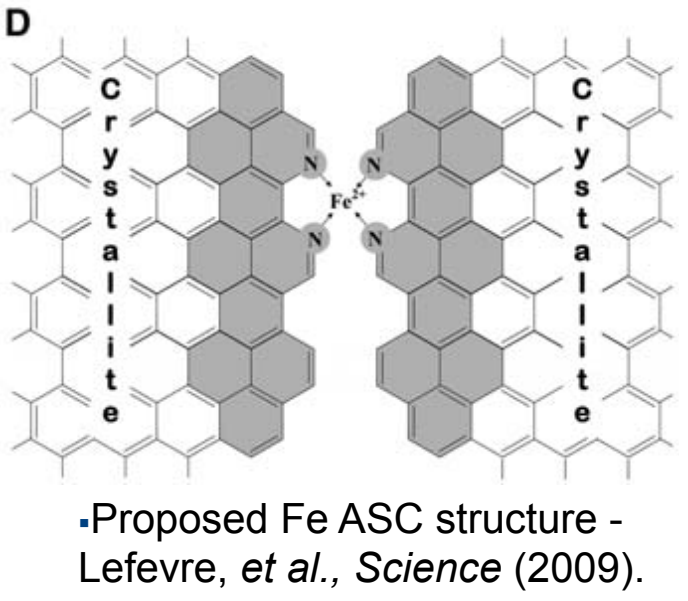

(a)

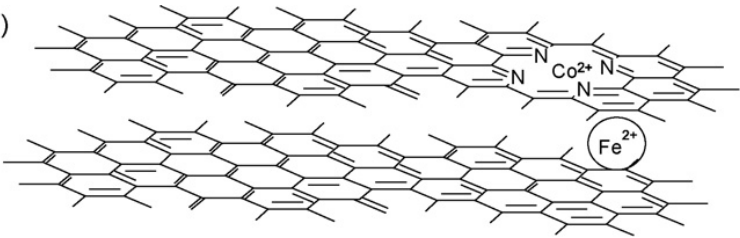

(b)
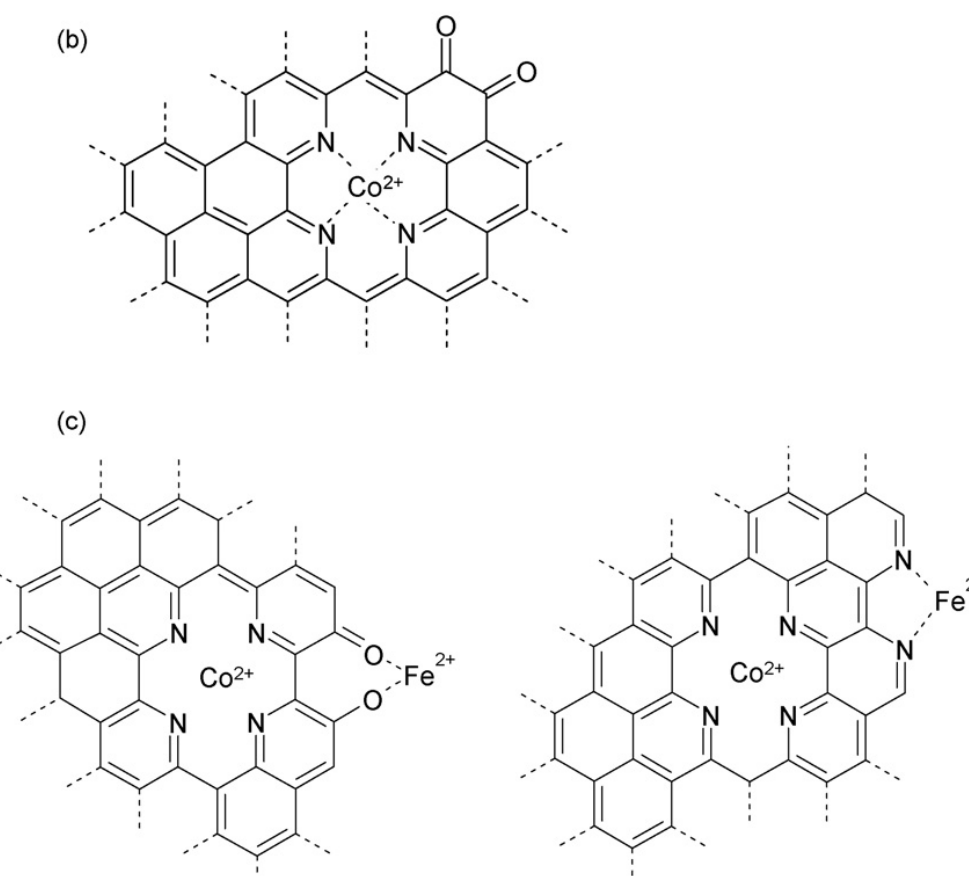

-Proposed M-N/C structures - Tributsch, et al., Electrochima Acta (2008). 


\section{Talk Outline}

- Background and Motivation

- Monte Carlo Search

- Insights from Density Functional Theory

- Vibrational spectra comparison with experiment

- Defected graphene nanoribbon stability

- Oxygen reduction reaction pathways

- Conclusions and Future Work 


\section{Monte Carlo Search Background}

- Large C/vacancy/N/Fe search space

- Need assumptions to model

- Activity increases with graphitization - use graphitic host material

- Relative concentrations

- Still many questions

- Defect Clustering?

- Edge vs. Center?

- Spin/Charge State?

- Structural Motifs?

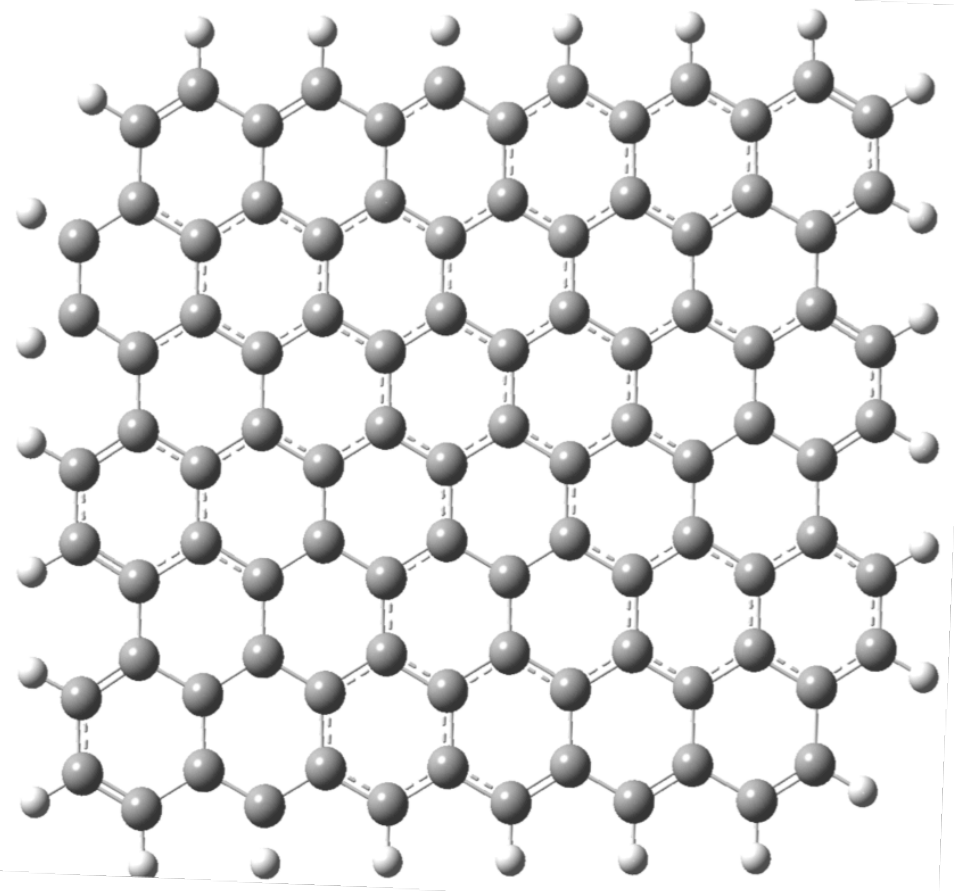

Holby, Wu, Zelenay and Taylor, ECS Transactions, in press 


\section{Monte Carlo Search Method}

- High throughput approach using semi-empirical (PM6) potential for fast screening

- Metropolis algorithm Monte Carlo (originally developed at LANL)

- Start w/ random structure

- Calculate energy (E1)

- Random swap

- Recalculate energy (E2)

- If E2<E1, accept swap

- If rand $<\exp \left[(E 1-E 2) / k_{B} T\right]$, accept swap

- Allows energy minimization and stochastic "uphill" swaps

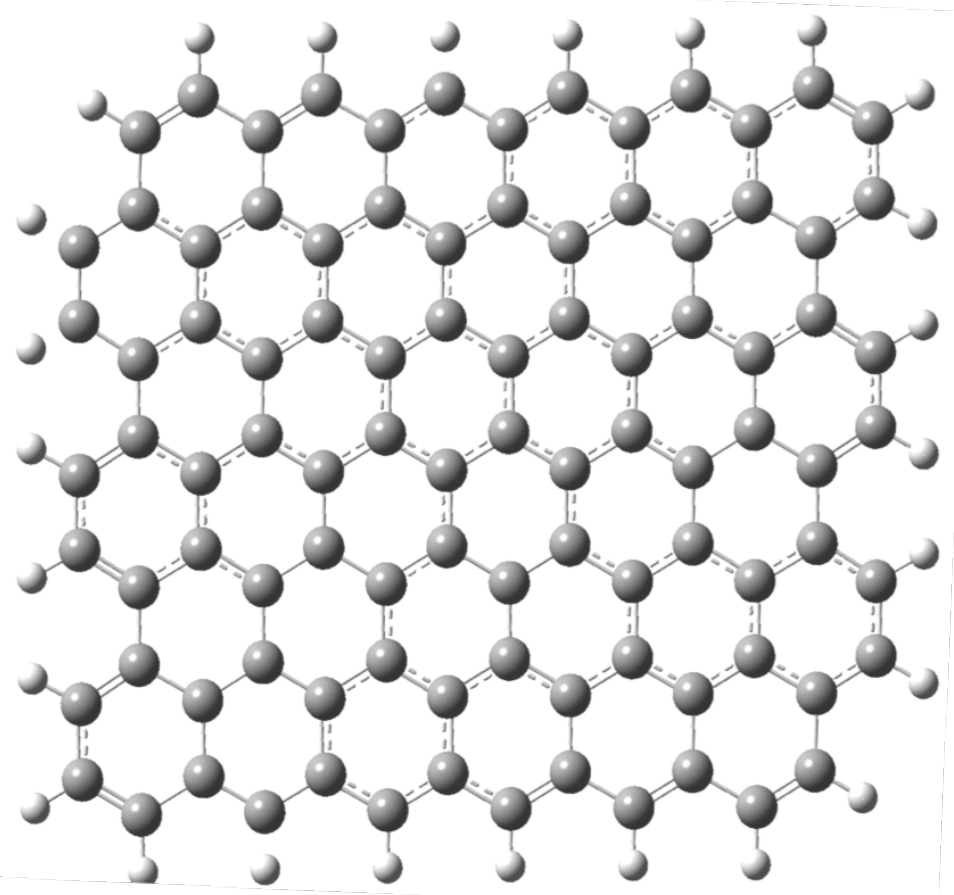

Holby, Wu, Zelenay and Taylor, ECS Transactions, in press 


\section{Monte Carlo Search Simulations}

- 800 initial structures

- 0-5 C vacancies

- Spread of spin and charge states

- 1000 iterations per structure

- Python wrapper with Gaussian '09 code

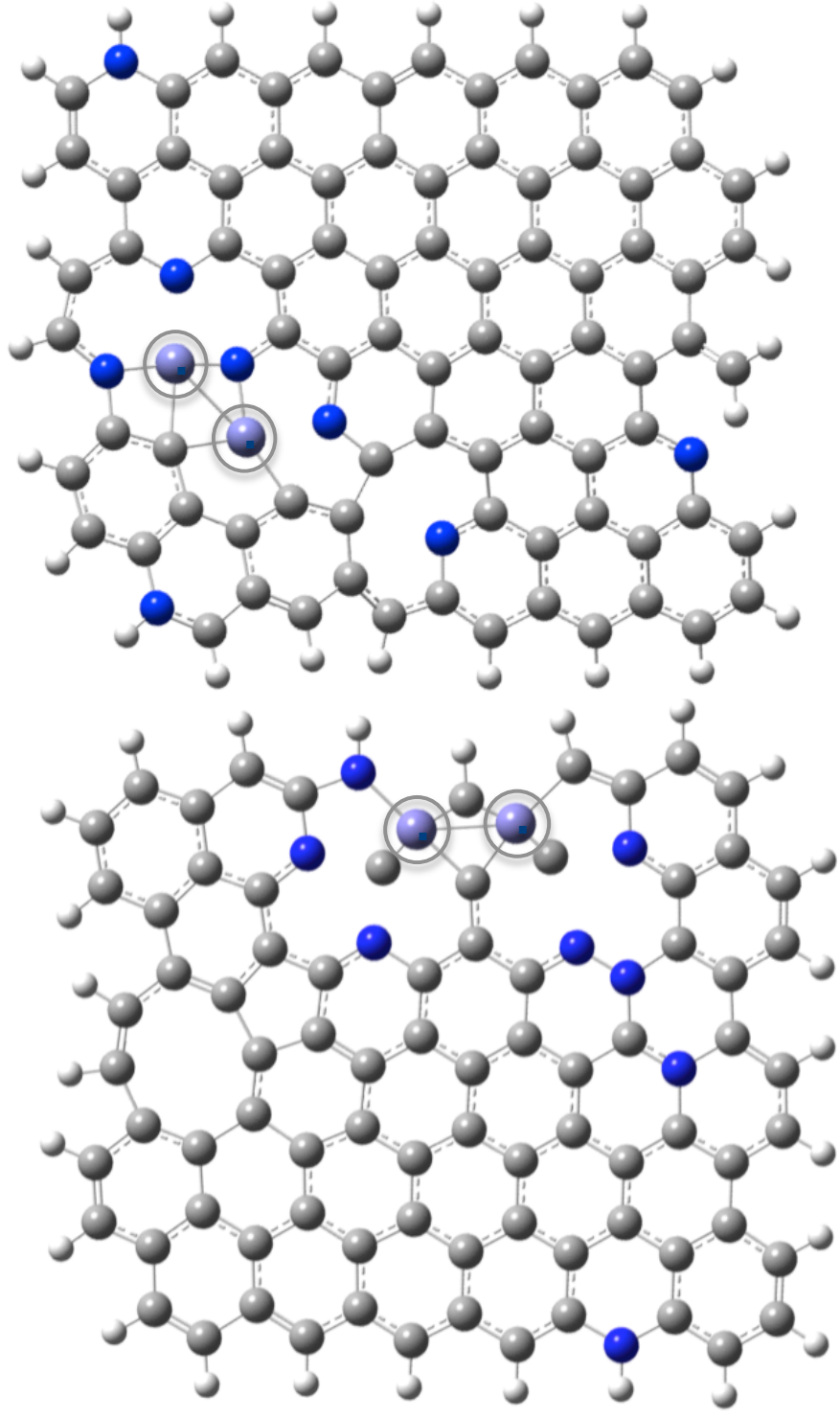

Holby, Wu, Zelenay and Taylor, ECS Transactions, in press 


\section{Monte Carlo Search Results}

- Proper vac. Structure

- Fe $2^{\text {nd }} \mathrm{NN}$ clustering with "bridge" atom

- $\mathrm{N}$ and vac. cluster with $\mathrm{Fe}$ structures

- Preference for edges vs. central positions

- Structure dominant over spin/ charge state

- Suggests motifs for higher level theory (DFT)

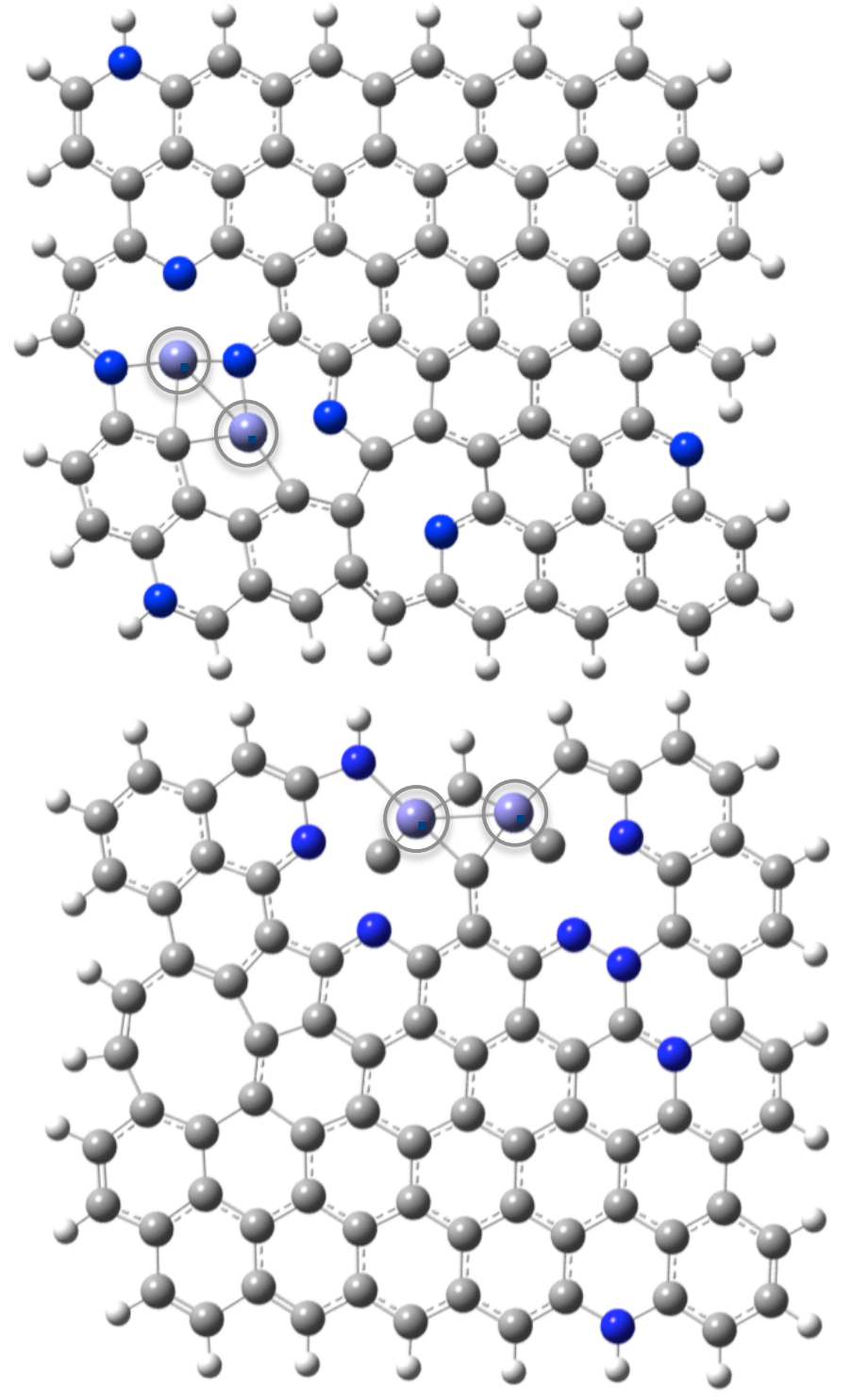

Holby, Wu, Zelenay and Taylor, ECS Transactions, in press 


\section{Talk Outline}

- Background and Motivation

- Monte Carlo Search

- Insights from Density Functional Theory

- Vibrational spectra comparison with experiment

- Defected graphene nanoribbon stability

- Oxygen reduction reaction pathways

- Conclusions and Future Work 


\section{DFT - Calculation Details}

- Vienna Ab-inito Simulation Package (VASP)

- PBE-GGA exchange and correlation functional

- $15 \AA$ vacuum between GNR images

- Converged to $<1 \mathrm{meV}$

- $400 \mathrm{eV}$ energy cutoff

- K point mesh Ax1x1 (GNR)

- Vibrational by force $(<0.02 \mathrm{eV} / \AA \AA)$

- MedeA for vibrational spectra

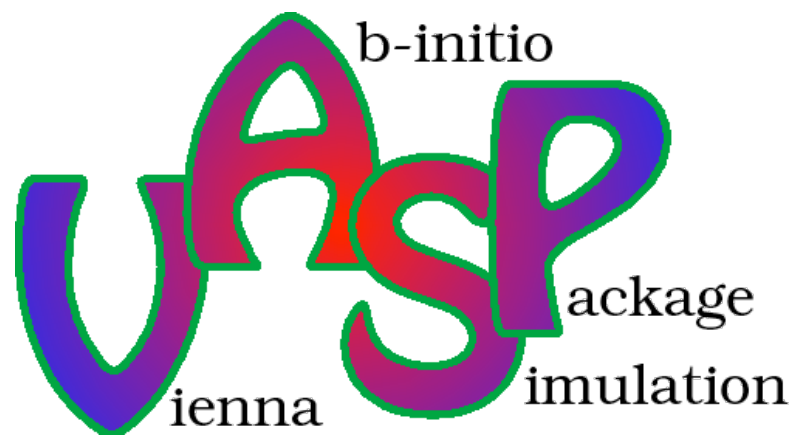

- Direct method - Parlinski, Li, and Kawazoe (PRL, 1997)

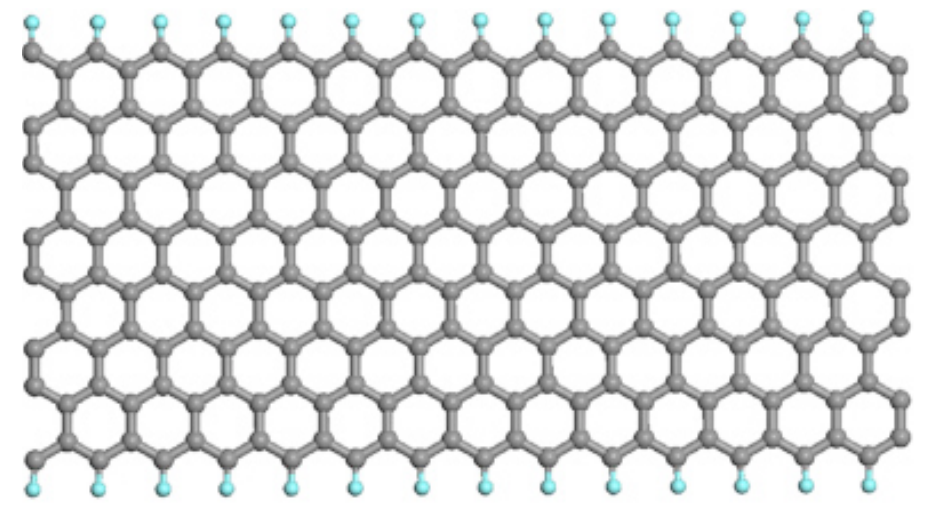




\section{Talk Outline}

- Background and Motivation

- Monte Carlo Search

- Insights from Density Functional Theory

- Vibrational spectra comparison with experiment

- Defected graphene nanoribbon stability

- Oxygen reduction reaction pathways

- Conclusions and Future Work 


\section{Vibrational Spectra and NRVS}

- Fe active or temple $\mathrm{C} / \mathrm{N}$ active site?

- Nuclear resonance vibrational spectroscopy (NRVS) with NO probe ligand $\left(\mathrm{O}_{2}\right.$ analog $)$ - first surface $\mathrm{Fe}$ specific spectroscopic technique (adapted from biology)

- DFT can calculate Fe spectra for direct comparison

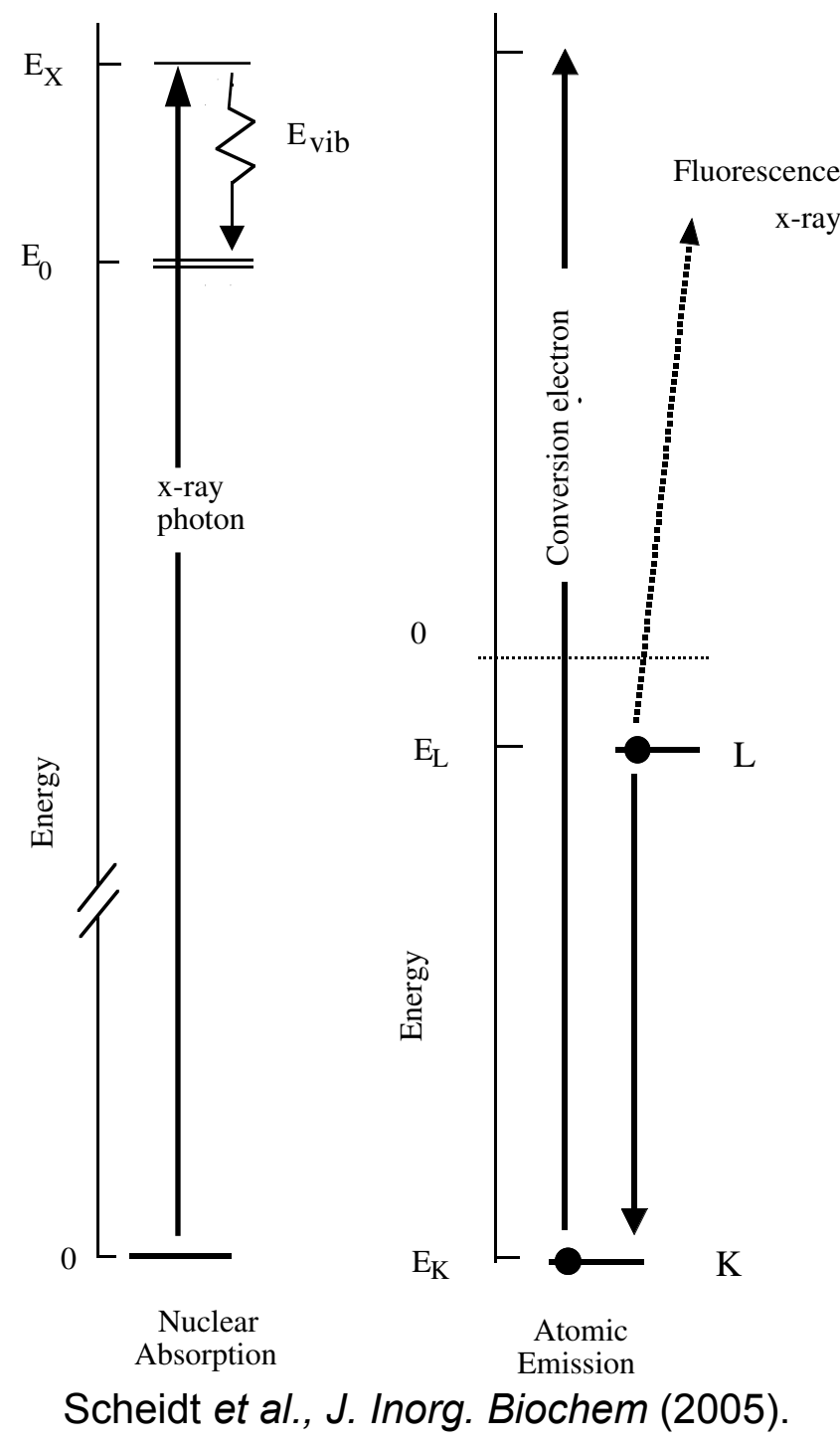




\section{NRVS - Findings}

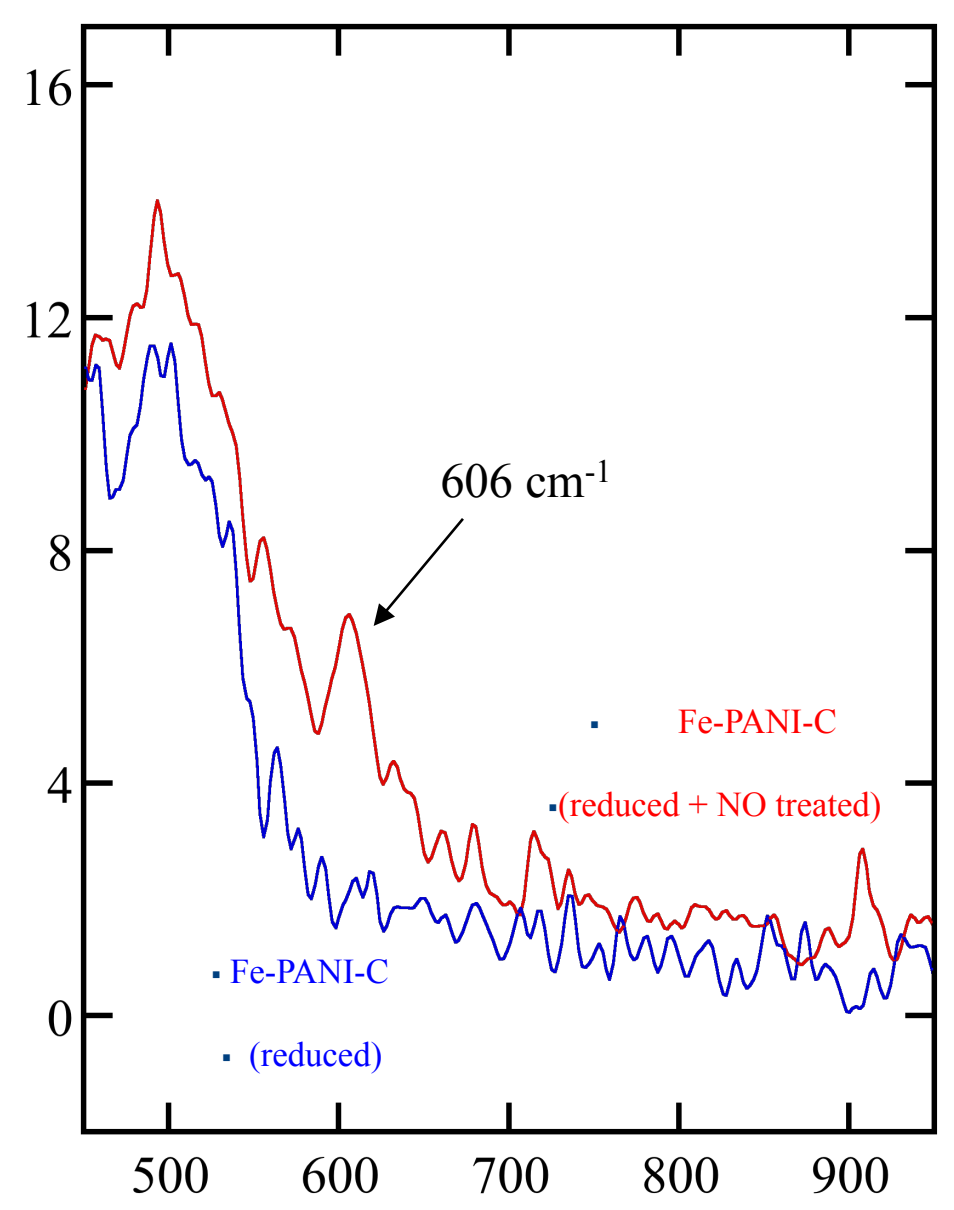

New Fe vibrations consistent with Fe$\mathrm{NO}$ ligation upon treatment with $\mathrm{NO}_{(\mathrm{g})}$ ?

Note: $\mathrm{NO}_{(\mathrm{g})}$ should only be able to access iron sites which are gas accessible (i.e. sites which are also accessible to $\mathrm{O}_{2}$ ) surface sensitive! 


\section{DFT - Vibrational Spectra}

- 3N-monovacancy edge site agrees with NRVS

- 4N-bivacancy edge site does not agree with NRVS

- Can discriminate atomic scale structure of sites!

- Fe coordination environment
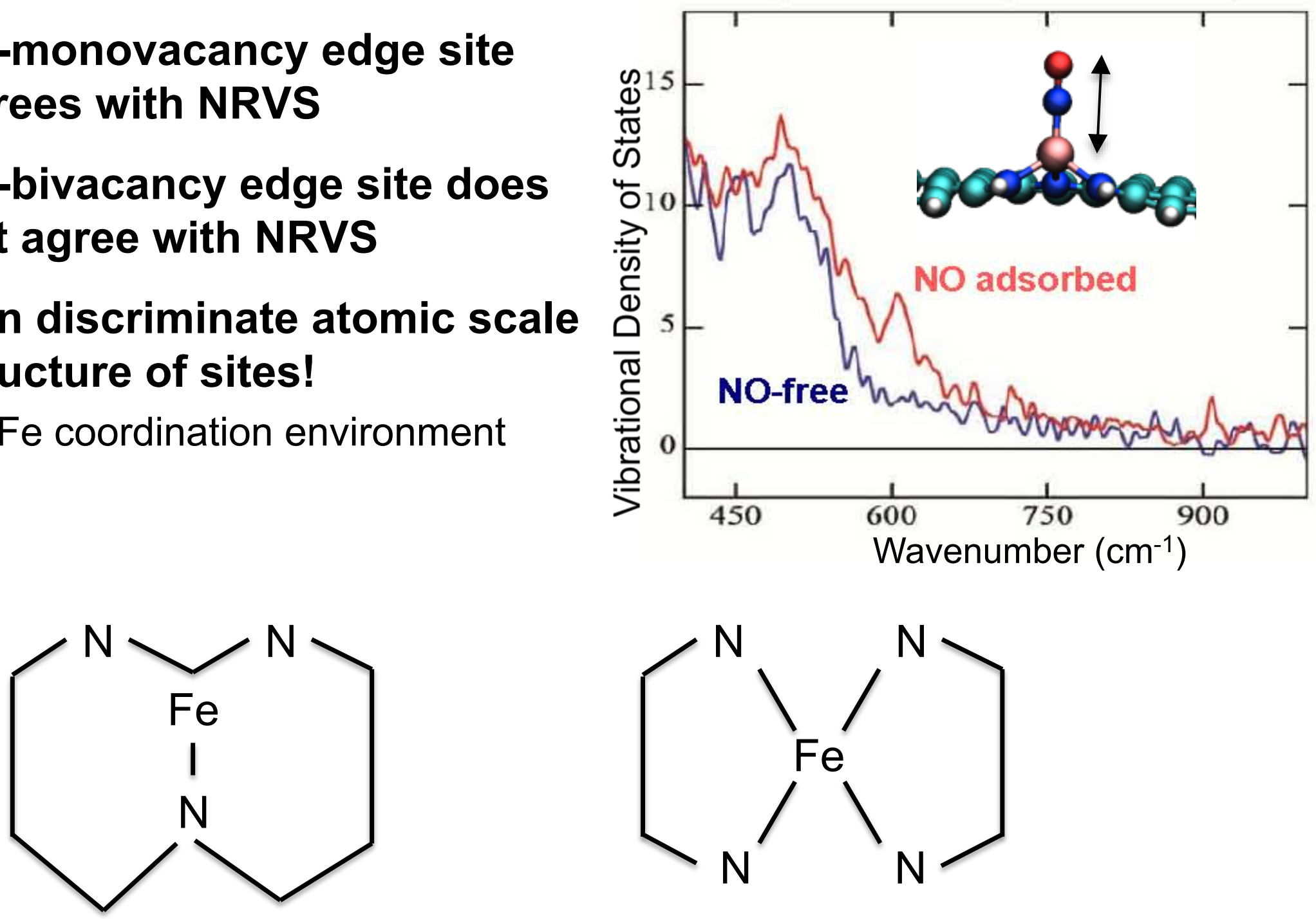


\section{Talk Outline}

- Background and Motivation

- Monte Carlo Search

- Insights from Density Functional Theory

- Vibrational spectra comparison with experiment

- Defected graphene nanoribbon stability

- Oxygen reduction reaction pathways

- Conclusions and Future Work 


\section{DFT - Graphene Nanoribbons}

- Use of zig-zag H-terminated graphene nanoribbon (GNR) host VASP code

- Comprehensive study of defects vs. position

- Defects Considered:

- Monovacancy $(\mathrm{a}, \mathrm{c})$

- 3N-monovacancy $(\mathrm{d}, \mathrm{f})$

- Fe/monovacancy (b)

- $\mathrm{Fe} / 3 \mathrm{~N}$-monovacancy (e)

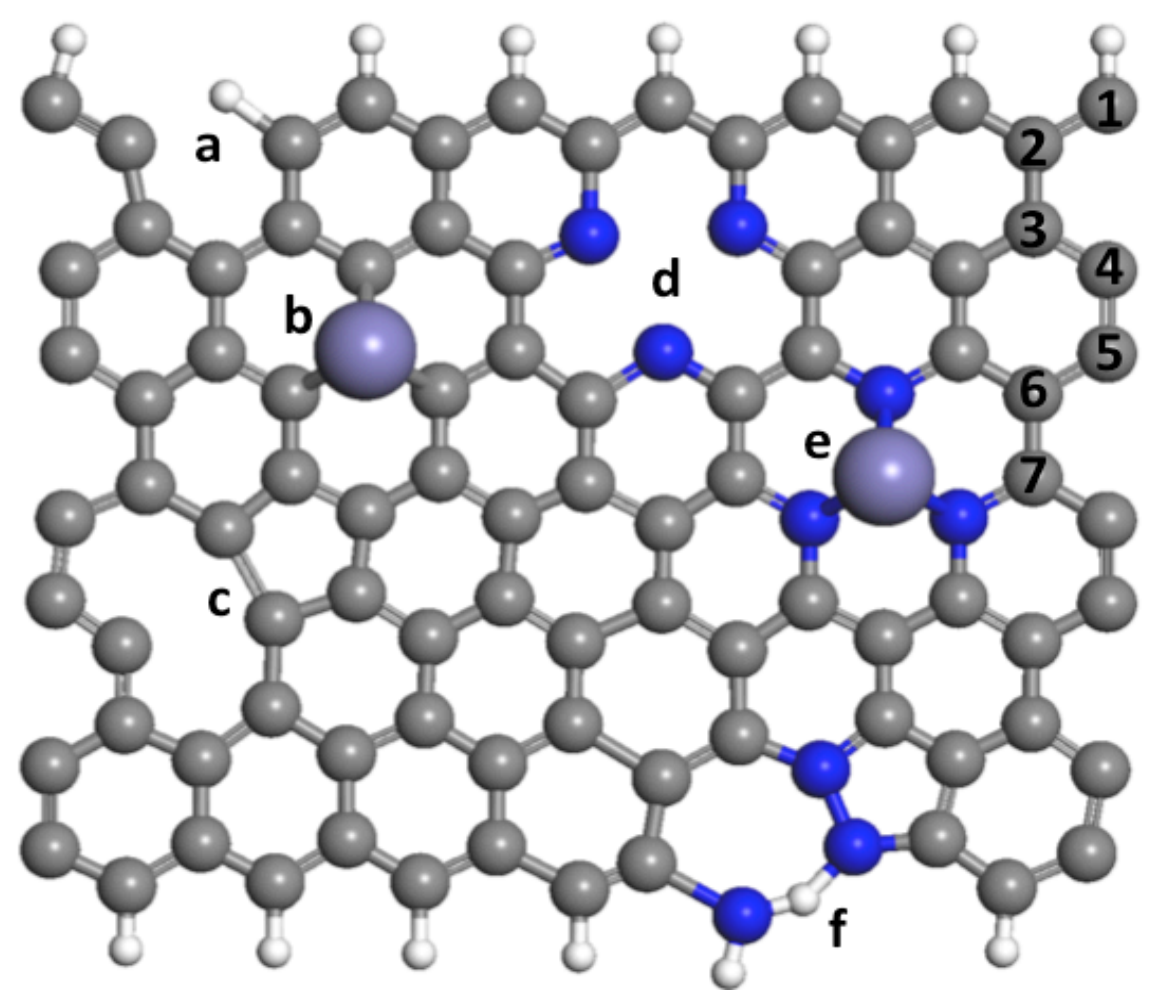

Holby and Taylor, App. Phys. Lett., (2012). 


\section{Graphene Nanoribbons -- Vacancies}

- Vacancy formation energy shows preference for $3 \mathrm{~N}$-termination (graphene / $\mathrm{N}_{2}$ ref. state)

- Non-monotonic behavior for 3N-monovacancy
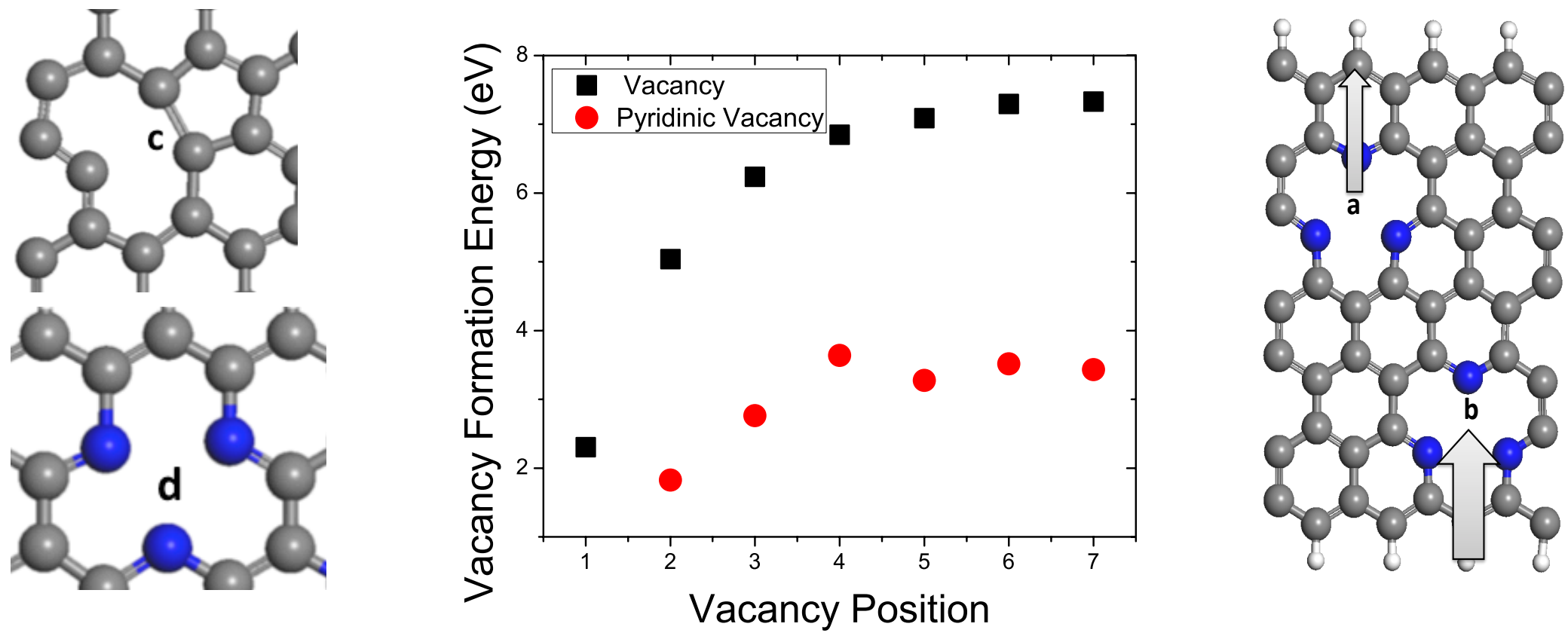

Holby and Taylor, App. Phys. Lett., (2012). 


\section{Graphene Nanoribbons - Fe and Vacancies}

- Fe/monovacancy has increasing formation energy going from edge to center but decreasing Fe binding

- If separate vacancy and Fe adsorption steps, Fe prefers to bind at center of GNR
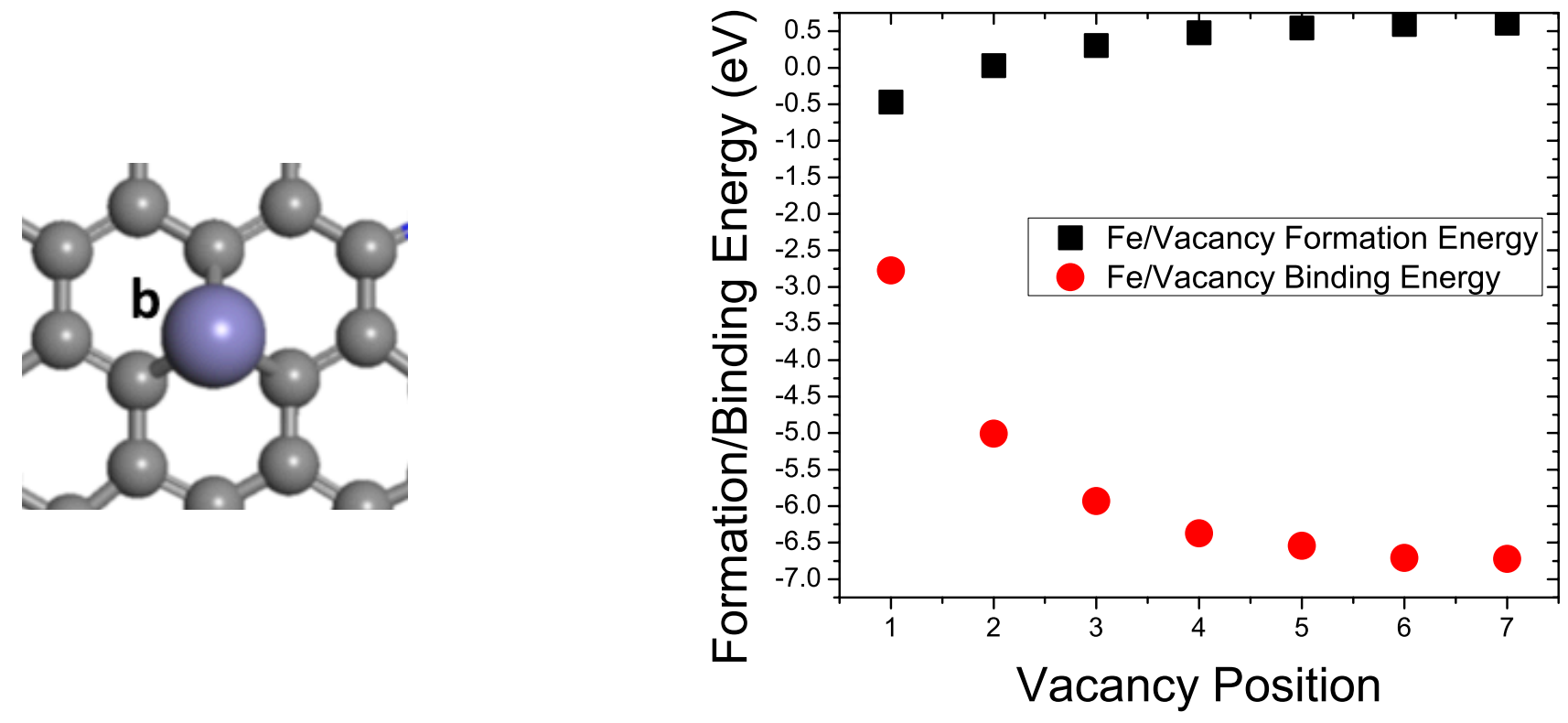

Holby and Taylor, App. Phys. Lett., (2012). 


\section{Graphene Nanoribbons - Fe, N, and Vacancies}

- Fe/3N-monovacancy has increasing formation energy going from edge to center and increasing Fe binding

- With N, Fe defects favorable at edge both from formation and binding
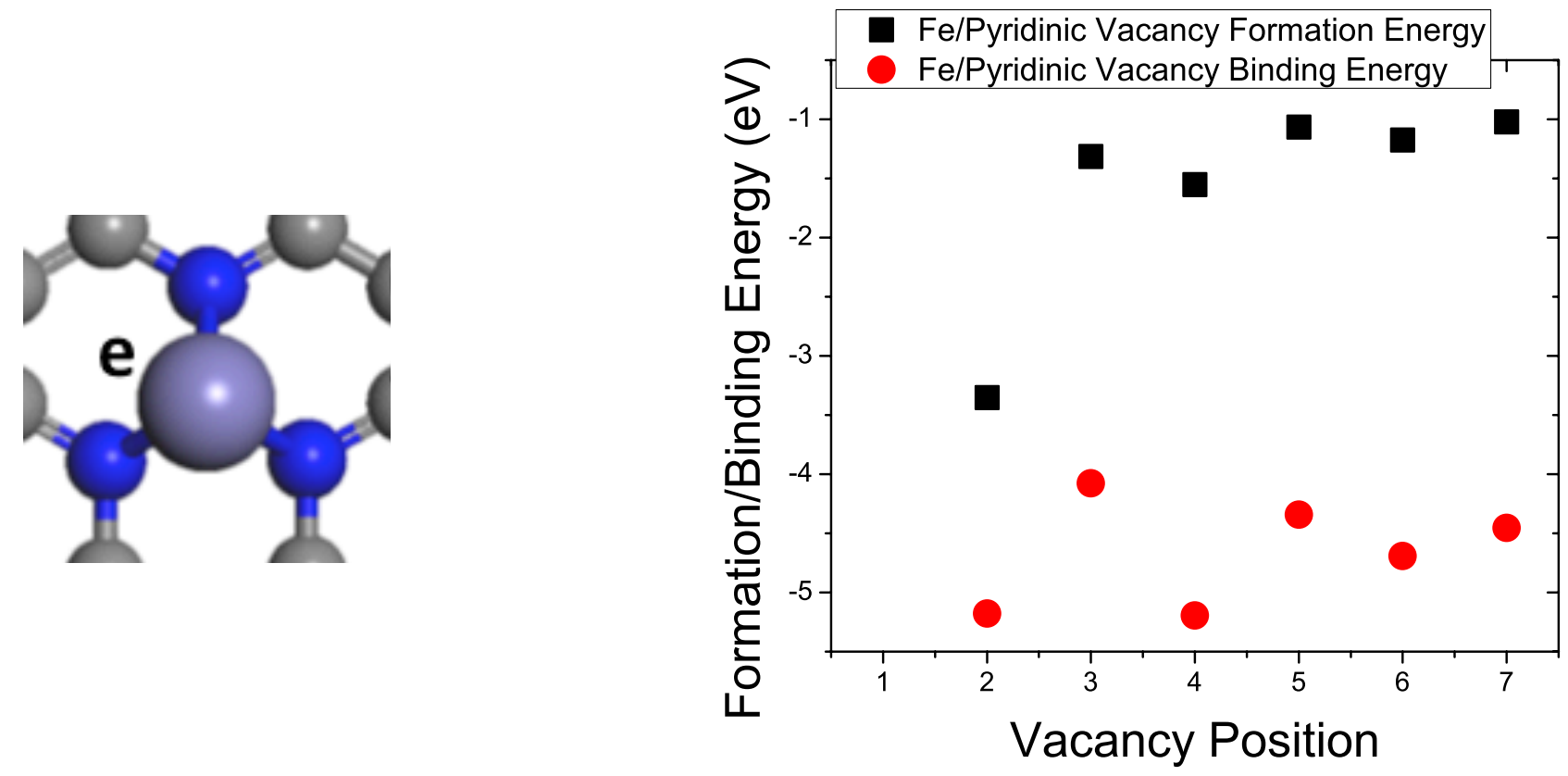

Holby and Taylor, App. Phys. Lett., (2012). 


\section{Graphene Nanoribbons -- Findings}

- Data suggests that $\mathbf{N}$ has two major effects relevant for catalysis:

- Decreased formation energy $\rightarrow$ More active sites

- Strong edge preference (despite formation pathway) means active sites at edges/increased accessibility

- Width of GNR (5-7) made little difference in formation and binding energies $(<75 \mathrm{meV})$

- Edge effects reach into GNR width (300 meV vacancy formation energy difference between center of GNR and "bulk" 2-D graphene) 


\section{DFT - Defect Clustering}

- Formation energies from DFT indicate clustering of $3 \mathrm{~N}$ monovacancy/Fe defects

- $5 \mathrm{~N}-2 \mathrm{Fe}$ most stable
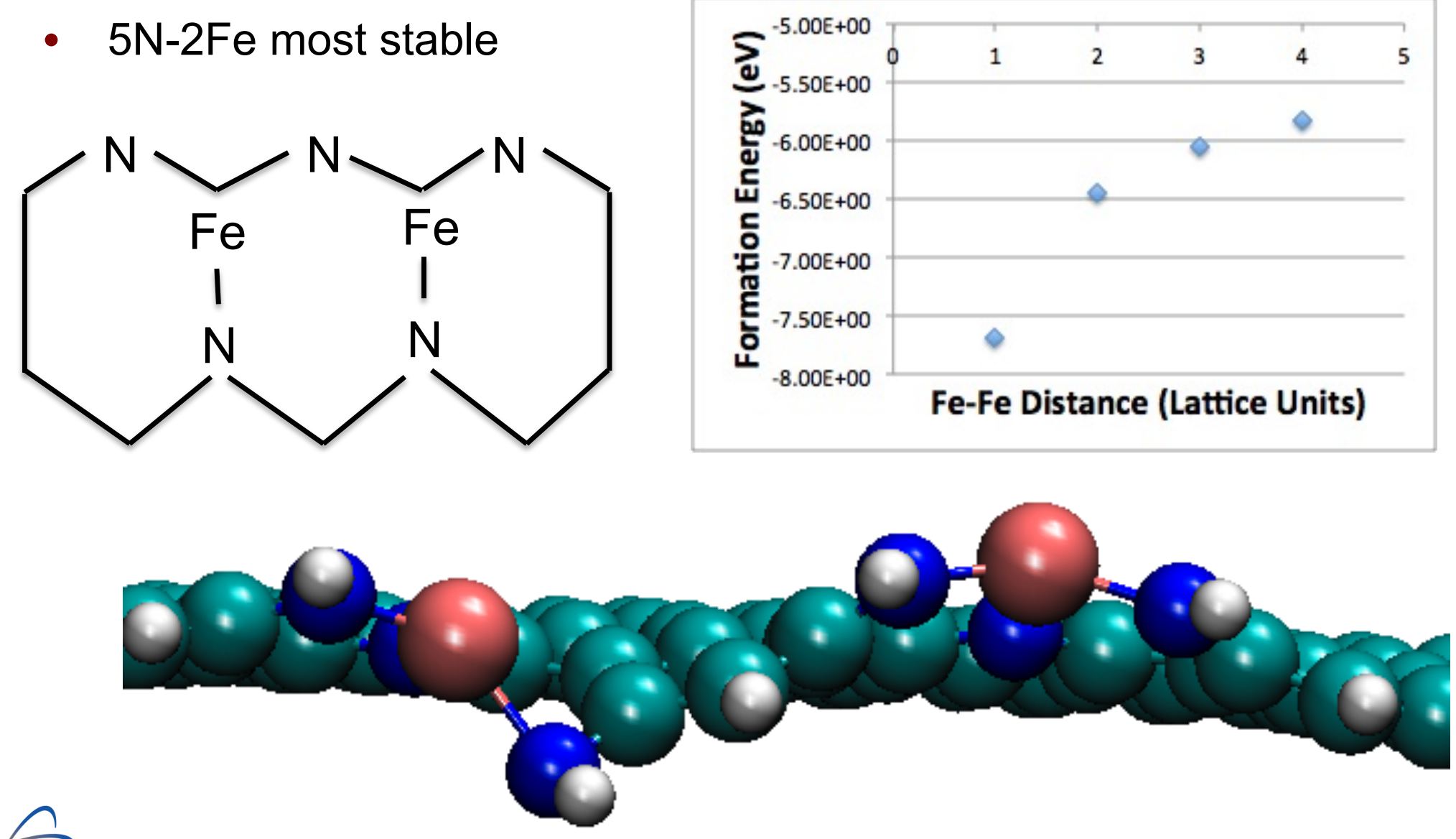


\section{Talk Outline}

- Background and Motivation

- Monte Carlo Search

- Insights from Density Functional Theory

- Vibrational spectra comparison with experiment

- Defected graphene nanoribbon stability

- Oxygen reduction reaction pathways

- Conclusions and Future Work 


\section{DFT - ORR Pathway}

- $4 \mathrm{e}^{-}$pathway $\rightarrow \mathrm{H}_{2} \mathrm{O}$ (desirable)

- 2 e- pathway $\rightarrow \mathrm{H}_{2} \mathrm{O}_{2}$ (undesirable)

- Multiple sub-pathways for 4 e- ORR

- "Dissociative Pathway": $\mathrm{O}_{2} \rightarrow 2 \mathrm{O}_{\text {ads }}$

- "Associative Pathway": $\mathrm{O}_{2} \rightarrow \mathrm{HOO}_{\text {ads }}$

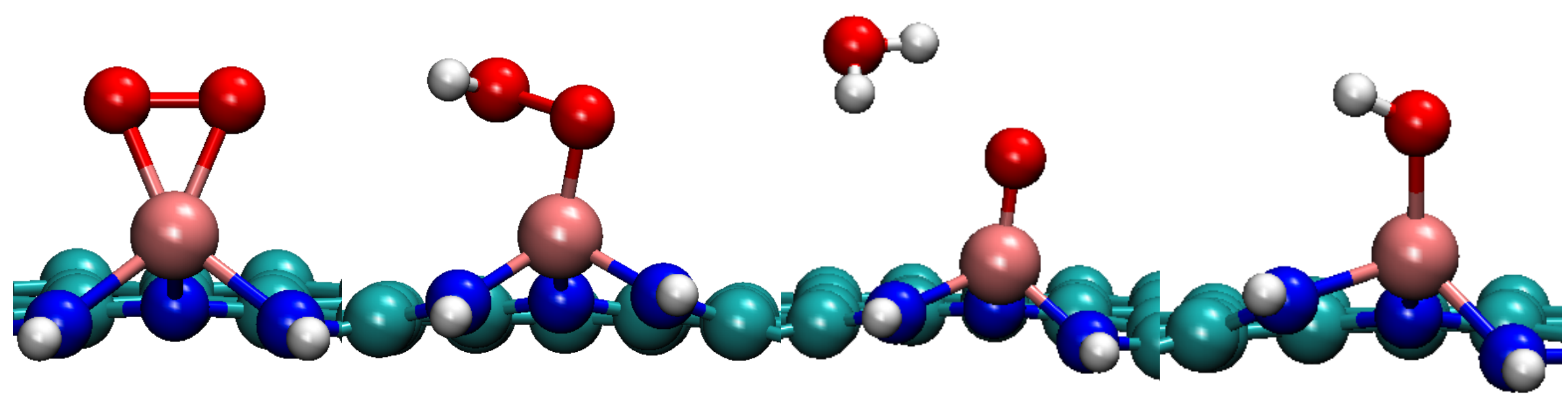




\section{DFT - ORR Pathway}

- Mono-Fe (3N-1Fe) GNR edge ASC follows associative pathway

- Multi-Fe (5N-2Fe) GNR edge ASC follows dissociative pathway

- Need more ASCS

- Requires more computational power

- Effect of solvation? Dispersion?

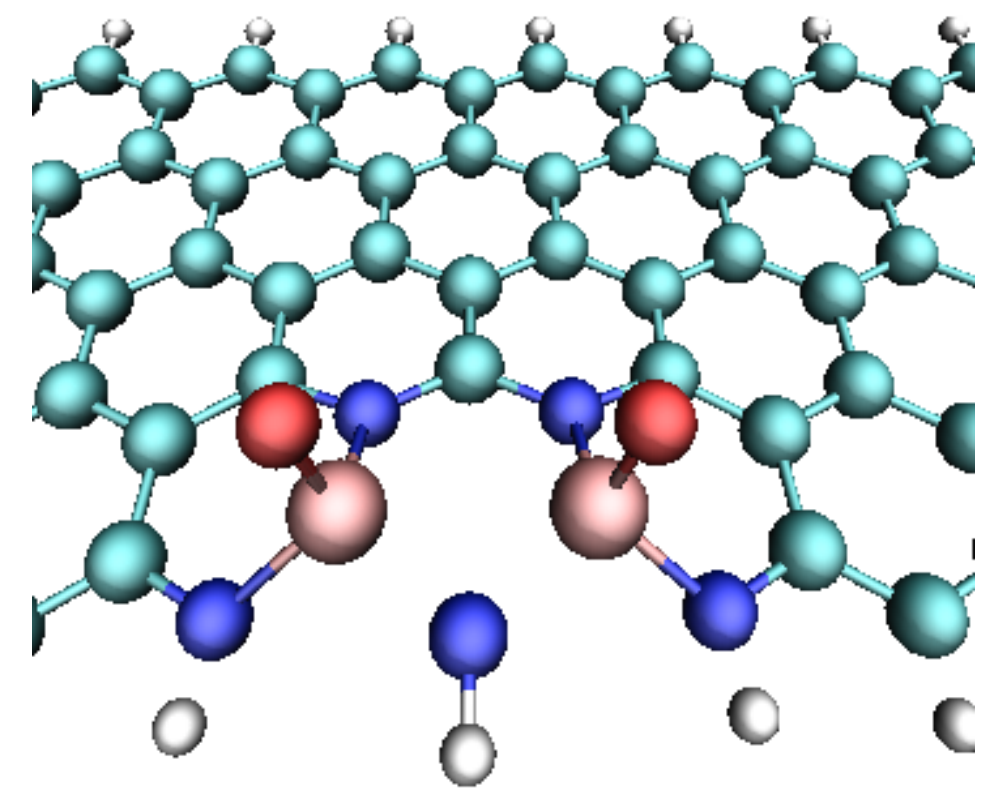




\section{DFT - ORR Pathway}

- Anderson and Nørskov Models of ORR suggests that $O$ binding energy of $\sim 2.38 \mathrm{eV}$ is ideal for dissociative pathway

- $5 \mathrm{~N}-2 \mathrm{Fe}$ site has binding energy of $2.41 \mathrm{eV}$

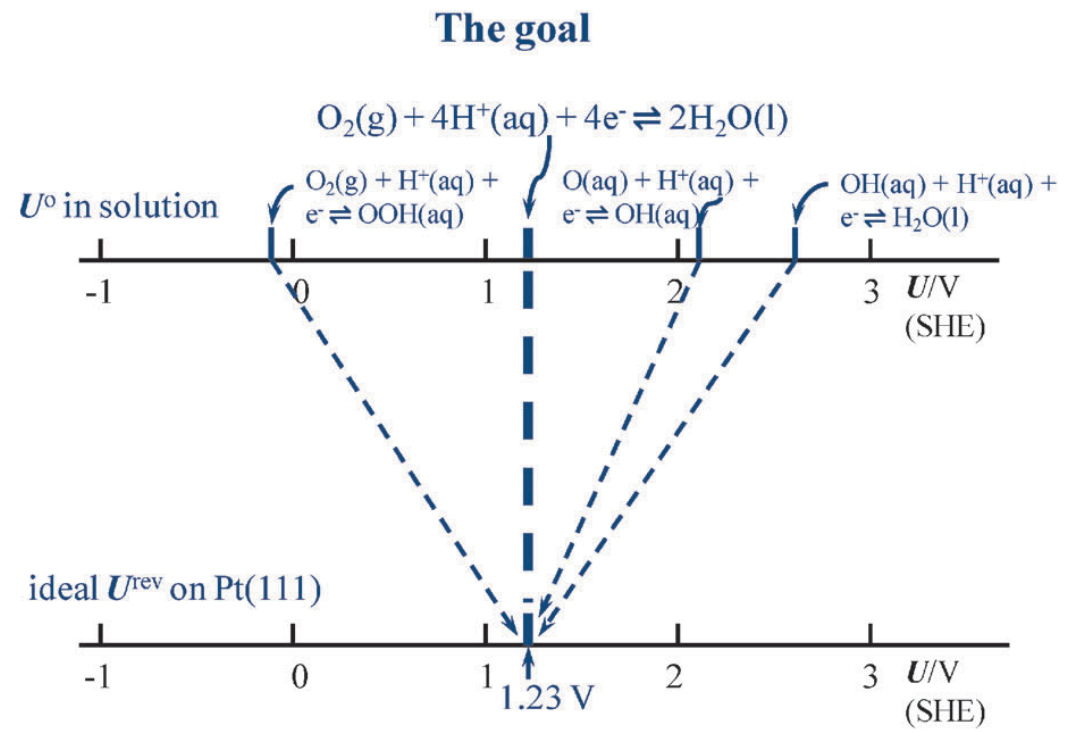

Anderson, Phys. Chem. Chem. Phys. (2012).

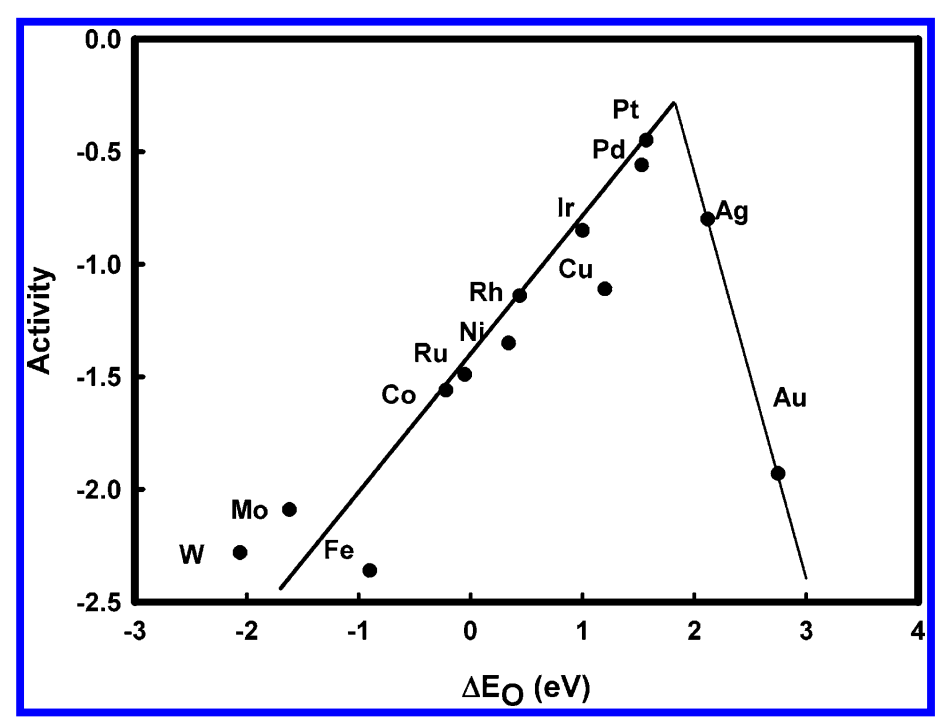

Norskov et al., J. Phys. Chem. B (2004). 


\section{Talk Outline}

- Background and Motivation

- Monte Carlo Search

- Insights from Density Functional Theory

- Defected graphene nanoribbon stability

- Vibrational spectra comparison with experiment

- Oxygen reduction reaction pathways

- Conclusions and Future Work 


\section{Conclusions}

- Spectroscopy/DFT (currently) point toward 3N coordinated Fe site vs. traditional $4 \mathrm{~N}$

- DFT/MC shows $3 \mathrm{~N}$ coordinated Fe sites are favored at GNR edge

- DFT/MC shows $3 \mathrm{~N}$ coordinated Fe sites cluster at GNR edge leading to $5 \mathrm{~N}-2 \mathrm{Fe}$ sites

- DFT shows $5 \mathrm{~N}-2 \mathrm{Fe}$ binding energy consistent with high activity and experimental spectra 


\section{Future Work}

- More ASCs!

- Vibrational spectra, formation energies, ORR pathways

(thermodynamics + kinetic barriers)

- Development of vibrational, stability, and activity descriptors what makes ASCs have certain properties?

- Synthesis targets based on stability, selectivity, and activity for rational design 


\section{Thank You!}

- Collaborators

- C. Taylor, P. Zelenay, G. Wu, H. Chung, M. Blair, LANL

- M. Neidig, Rochester

- Funding

- LDRD-ER

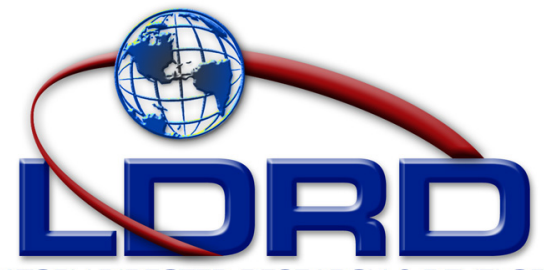

LABORATORY DIRECTED RESEARCH \& DEVELOPMENT

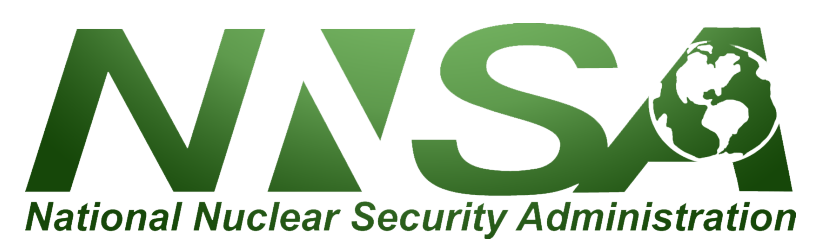

National Nuclear Security Administration

- Computation Resources

- LANL IC

- ANL CNM

- XSEDE Network
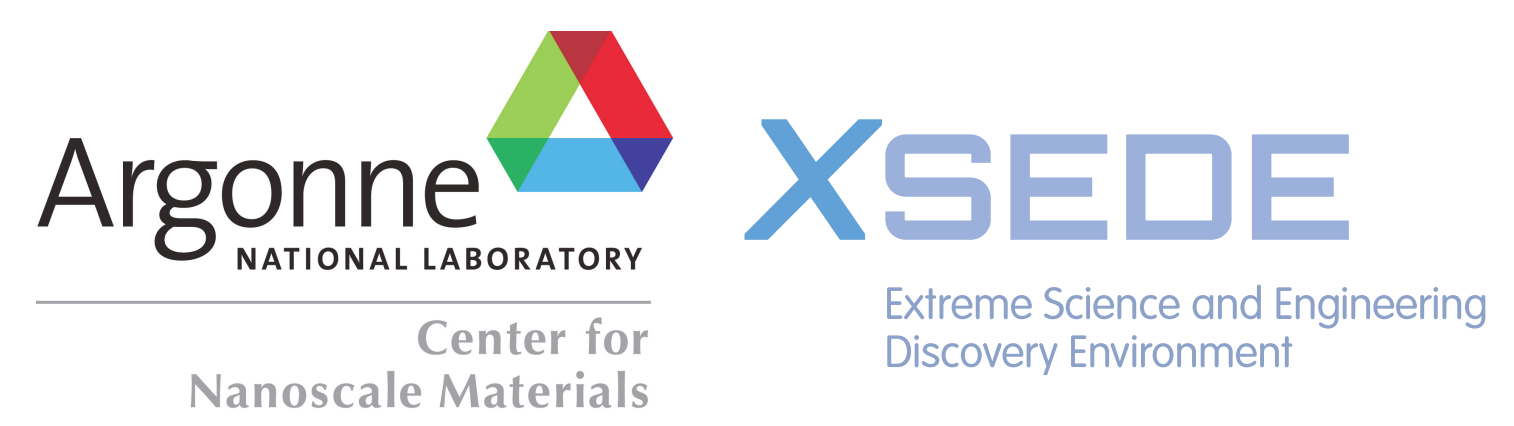